\title{
A novel monoclonal antibody efficiently blocks the infection of serotype 4 fowl adenovirus by targeting fiber- 2
}

\author{
Ping Wang ${ }^{1,2,3 \dagger}$, Jianjun Zhang ${ }^{4 \dagger}$, Weikang Wang 1,2,3 , Tuofan Li 1,2,3, Guangchen Liang ${ }^{1,2,3}$, Hongxia Shao 1,2,3,4,5 \\ Wei Gao ${ }^{1,2,3,4,5}$, Aijian Qin 1,2,3,4,5* and Jianqiang Ye ${ }^{1,2,3,4,5^{*}}$
}

\begin{abstract}
A recent outbreak of hepatitis-hydropericardium syndrome caused by serotype 4 fowl adenovirus (FAdV-4) has resulted in significant economic losses to the poultry industry worldwide. However, little is known about the molecular pathogenesis of FAdV-4. In this study, a novel monoclonal antibody (mAb) targeting the fiber-2 protein of FAdV-4 was generated, mAb 3C2. Indirect immunofluorescence assay showed that mAb 3C2 neither reacted with serotype 8 fowl adenovirus (FAdV-8) nor reacted with the fiber-1 protein of FAdV-4; it specifically reacted with the fiber-2 protein of FAdV-4. Notably, mAb 3C2 could efficiently immunoprecipitate the fiber-2 protein in chicken liver cells either infected with FAdV-4 or transfected with pcDNA3.1-Fiber2. Moreover, mAb 3C2 demonstrated marked neutralizing activity against FAdV-4 and could efficiently inhibit the infection of FAdV-4 in vitro. Using truncated fiber-2 constructs, the epitope recognized by mAb 3C2 was determined to be located between amino acids 416-448 at the C-terminus of fiber-2. Our data not only provide a foundation for the establishment of a rapid fiber-2 peptide-based diagnostic assay for FAdV-4 but also highlight the critical role of the fiber-2 protein in mediating infection by FAdV-4. Furthermore, the epitope recognized by 3C2 might serve as a novel target for the development of a vaccine targeting FAdV-4.
\end{abstract}

\section{Introduction}

Fowl adenovirus (FAdV) is a member of the family Adenoviridae, genus Aviadenovirus [1]. To date, five species (FAdV-A to FAdV-E) and 12 serotypes (FAdV-1 to $8 \mathrm{a}$ and $8 \mathrm{~b}$ to 11 ) of FAdV have been identified by cross-neutralization assay and genome analysis [2]. FAdV infection generally causes subclinical symptoms in infected chickens; however, acute infections can result in inclusion body hepatitis (IBH), hepatitis-hydropericardium syndrome (HPS), and gizzard erosion and ulceration (GEU) [2-4]. Among the 12 serotypes, FAdV-2, 8a, 8b and 11 generally cause IBH, whereas FAdV-4 is the main cause

\footnotetext{
*Correspondence: aijian@yzu.edu.cn; jqye@yzu.edu.cn

${ }^{\dagger}$ Ping Wang and Jianjun Zhang contributed equally to this manuscript

${ }^{1}$ Key Laboratory of Jiangsu Preventive Veterinary Medicine, Key

Laboratory for Avian Preventive Medicine, Ministry of Education, College

of Veterinary Medicine, Yangzhou University, Yangzhou 225009, Jiangsu,

China

Full list of author information is available at the end of the article
}

of endemic HPS [5-8]. Notably, HPS caused by FAdV-4 is a major killer in chicken flocks [9]. Recently, an outbreak of FAdV-4 reached epidemic proportions, possibly due to enhanced virulence, causing massive economic losses in the poultry industry [2, 3, 6, 10-13]. Notably, the mortality caused by endemic FAdV-4 in China has reached as high as $80 \%$ in several domestic chicken flocks [13-16]. Moreover, sequencing analysis revealed that recent Chinese FAdV-4 isolates carried unique mutations with significant deletions in their genome compared with FAdV-4 isolates from other countries and regions [13, 14]. However, little is known about the molecular mechanism underlying the infection and pathogenesis of FAdV-4.

Among the structural proteins encoded by adenovirus, the fiber protein plays vital roles in mediating viral infection and determining the antigenicity [17-19]. In contrast to other adenoviruses, FAdV-1, FAdV-4 and FAdV-10 encode two fiber proteins, designated fiber-1 and fiber-2 [17]. Although a previous report showed 
that recombinant fiber- 2 protein could provide better protection against FAdV-4 than recombinant fiber-1 [20], the roles of the two fiber proteins in the infection and pathogenesis of FAdV-4 are unclear. Due to the lack of any monoclonal antibody $(\mathrm{mAb})$ specific for the fiber proteins of FAdV-4, the progression of such studies has been severely limited. In this study, a novel $\mathrm{mAb}$ specific to the fiber- 2 protein of FAdV-4 (designated mAb $3 \mathrm{C} 2$ ) was generated, and its epitope was identified. mAb $3 \mathrm{C} 2$ could not only immunoprecipitate the fiber- 2 protein in infected cells but also blocked the infection of FAdV-4 in vitro.

\section{Materials and methods}

\section{Viruses, cells and plasmids}

FAdV-4 isolate SD2015 and FAdV-8 isolate SQ2015 were isolated and maintained in our laboratory [13]. The chicken liver cell line (LMH) was purchased from ATCC and cultured in F12/DMEM (Gibco, NY, USA) supplemented with 10\% FBS (Lonsera, Shanghai, China). Plasmids pcDNA3.1-F1 and pcDNA3.1-F2 expressing fiber-1 and fiber-2 of FAdV-4, respectively, were generated in our laboratory.

\section{Antibodies}

Chicken sera against FAdV-4 and FAdV-8 were generated through vaccination of 14-day-old SPF chickens with the corresponding inactivated viruses, provided by Sinopharm Yangzhou VAC Biological Engineering Co., Ltd. Monoclonal antibody 6E6 against HA of H9N2 was developed in our laboratory [21]. FITC-labelled goat anti-mouse IgG, HRP-labelled goat anti-mouse IgG and HRP-labelled rabbit anti-chicken $\operatorname{IgY}(\mathrm{H}+\mathrm{L})$ were purchased from Sigma (CA, USA).

\section{Generation of mAbs}

$10^{7} \mathrm{TCID}_{50}$ of FAdV-4 isolate SD2015 was used to immunize 6-week-old BALB/C mice four times every 10 days. At day 4 following the fourth immunization, the splenic cells from one immunized mouse were collected and fused with SP2/0 cells with PEG1500 (Roche, Mannheim, Germany), as previously described [22]. After culture with HAT-selective medium (Sigma), hybridoma cells secreting antibodies against the fiber-2 protein of FAdV-4 were screened using ELISA coating with purified GSTFiber2 (generated in our laboratory). After sub-cloning of the positive hybridoma cells, the characteristics of mAbs secreted by these positive clones were identified through immunofluorescence assay, ELISA, immunoprecipitation and neutralization testing. The isotype of $\mathrm{mAb}$ was determined with a mouse mAb isotyping kit (Thermo Scientific, Massachusetts, USA) according to the manufacturer's protocol. The mAbs in ascites were generated as previously described and purified using protein $\mathrm{G}$ columns (GE Healthcare Life sciences, Uppsala, Sweden) [23].

\section{ELISA}

The purified GST-Fiber2 protein (generated in our laboratory) was used to coat the wells of plates $[0.36 \mu \mathrm{g}$ protein in each well) at $4{ }^{\circ} \mathrm{C}$ overnight. The ELISA plates were washed with PBST (0.01 M phosphate-buffered saline (PBS), $\mathrm{pH} 7.2,0.05 \%$ Tween 20] once and then blocked with PBST containing 5\% skim milk for $1 \mathrm{~h}$. After washing once, the supernatant of the hybridoma cells or serial dilutions of mAbs were added and incubated for $1 \mathrm{~h}$ at $37^{\circ} \mathrm{C}$. After three washes with PBST, the plates were supplemented with $100 \mu \mathrm{L}$ of a 1:10 000 dilution of HRPlabelled goat anti-mouse IgG. After $30 \mathrm{~min}$ at $37^{\circ} \mathrm{C}$, the ELISA plates were washed three times with PBST, and then supplemented with $100 \mu \mathrm{L}$ TMB (Solarbio, Beijing, China) for development. After $10 \mathrm{~min}$ at $37^{\circ} \mathrm{C}$, the development reaction was stopped by adding $50 \mu \mathrm{L}$ of $2 \mathrm{M}$ $\mathrm{H}_{2} \mathrm{SO}_{4}$, and the absorbance values at $450 \mathrm{~nm}$ (OD450) were measured with a Microplate Reader (TECAN Infinite M200 Pro). When the OD450 value of the sample was greater than 0.2 , the sample was regarded as positive.

\section{Indirect immunofluorescence assay (IFA)}

LMH cells were infected with 1000 TCID $_{50}$ of FAdV-4 SD2015 and FAdV-8 SQ2015 or transfected with pcDNA3.1-F1 and pcDNA3.1-F2, respectively. After day 3 post-infection or transfection, the cells were fixed with chilled acetone and ethanol (3:2) for $5 \mathrm{~min}$. mAbs at the indicated dilutions were incubated with the fixed cells for $45 \mathrm{~min}$. After three washes with PBS, FITC-conjugated secondary antibodies at 1:150 dilutions were incubated with the cells for another $45 \mathrm{~min}$. After three washes with PBS, the plates were observed with an inverted fluorescence microscope.

\section{Immunoprecipitation and immunoblotting}

LMH cells infected with 1000 TCID $_{50}$ of FAdV-4 SD2015 or transfected with pcDNA3.1-F2 for 3 days were collected and lysed in lysis buffer (CST, MA, USA) with PMSF (Beyotime, Shanghai China), protease and phosphatase inhibitors (CST). Lysates were then mixed and immunoprecipitated with $2 \mu \mathrm{g} \mathrm{mAb}$ at $4{ }^{\circ} \mathrm{C}$ overnight. The next day, $40 \mu \mathrm{L}$ of protein G-Sepharose beads (Beyotime) were added to the mixture and incubated at $4{ }^{\circ} \mathrm{C}$ for $3 \mathrm{~h}$. Then, the mixture of proteins and beads was washed five times with PBS, and the immunoprecipitated proteins were eluted by boiling with loading buffer. After separation via SDS-PAGE, the denatured samples were transferred onto nitrocellulose membranes (NCs) (GE Healthcare Life sciences, Freiburg, Germany) for Western 
Table 1 Primers for amplifying the truncated fiber2 gene and the linear pCDNA3.1

\begin{tabular}{ll}
\hline PCR product & Primer sequence (5'-3') \\
\hline F2_1-415aa & Forward: AGCTTGGTACCGAATGCTCCGGGCCCCTAA \\
& Reverse: ATATCTGCAGAATTACTATAGCATAGAAG \\
F2_1-382aa & Forward: AGCTTGGTACCGAATGCTCCGGGCCCCTAA \\
& Reverse: ATATCTGCAGAATTAGGCTGAACACTTGG \\
F2_1-349aa & Forward: AGCTTGGTACCGAATGCTCCGGGCCCCTAA \\
& Reverse: ATATCTGCAGAATTTACGTGAGGGTGGGG \\
F2_1-316aa & Forward: AGCTTGGTACCGAATGCTCCGGGCCCTAA \\
& Reverse: ATATCTGCAGAATTTATTGGCGGAGTTGAG \\
F2_1-283aa & Forward: AGCTTGGTACCGAATGCTCCGGGCCCCTAA \\
& Reverse: ATATCTGCAGAATTTAGGTAGTAGGCGCAA \\
F2_1-250aa & Forward: AGCTTGGTACCGAATGCTCCGGGCCCCTAA \\
& Reverse: ATATCTGCAGAATTTAGGAGACGCTCCCCC \\
F2_1-217aa & Forward: AGCTTGGTACCGAATGCTCCGGGCCCCTAA \\
& Reverse: ATATCTGCAGAATTAGGTCCGCTGGGATC \\
F2_1-182aa & Forward: AGCTTGGTACCGAATGCTCCGGGCCCCTAA \\
& Reverse: ATATCTGCAGAATTTACCTGCGCTTTTAGG \\
Linear pcDNA3.1 & Forward: GAATTCTGCAGATATCCAGCACAGTG \\
& Reverse: GCTCGGTACCAAGCTTAAGTTAAACG \\
\hline
\end{tabular}

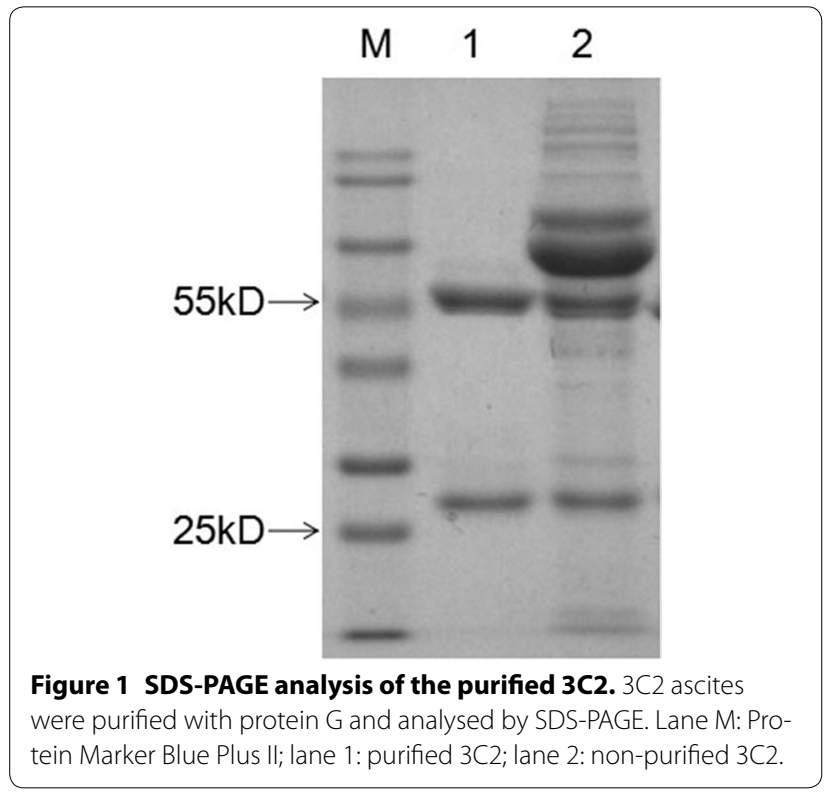

blot analysis. After blocking with skim milk in PBST for $1 \mathrm{~h}$ at room temperature (RT), the NCs were incubated with chicken sera against FAdV-4 (1:1000 dilution in PBST) for $2 \mathrm{~h}$ at RT. After three washes with PBST, the $\mathrm{NCs}$ were incubated with HRP-conjugated rabbit antichicken IgG (1:10 000 dilution in PBST). After three washes, the NCs were developed using a fully automatic chemiluminescence image analysis system (Tanon 5200).

\section{Neutralization testing}

Serial dilutions of mAbs were first mixed with 1000 TCID $_{50}$ of FAdV-4 isolate SD2015 in equal volumes and incubated for $1 \mathrm{~h}$ at $37{ }^{\circ} \mathrm{C}$. Then, the mixture was inoculated into fresh LMH cells. After incubation for $2 \mathrm{~h}$, the inoculated supernatant was discarded, and the inoculated cells were washed once with PBS. The inoculated cells were then maintained in DMEM/F12 medium with $1 \%$ FBS for 3 days. Then, the viral replication in these inoculated cells was analysed through IFA and Western blot, as described above.

\section{Epitope mapping}

To map the epitopes recognized by mAb $3 \mathrm{C} 2$, serial fiber- 2 recombinant constructs with various deletions at the C-terminus were generated with a ClonExpress II One Step Cloning Kit (Vazyme Biotech, Nanjing, China) as previously described. The primers used for amplifying the fiber-2 gene with different deletions and the linear pcDNA3.1 vector are listed in Table 1 . LMH cells transfected with the different fiber- 2 recombinants were fixed at day 2 post-transfection and stained with mAb $3 \mathrm{C} 2$ through IFA, as described above.

\section{Results}

Generation of a novel monoclonal antibody against fiber-2 of FAdV-4

To generate monoclonal antibodies specific to fiber-2 of FAdV-4, FAdV-4 isolate SD2015 was used to immunize BALB/C mice. After the fusion of splenic cells from immunized mice and SP2/0 cells, hybridoma cells secreting antibodies against the fiber- 2 protein of FAdV-4 were screened by ELISA. In the ELISA, purified GST-Fiber2 was used as the coating antigen. Ultimately, one hybridoma clone showed strong positivity in ELISA screening and was designated $\mathrm{mAb} 3 \mathrm{C} 2$. After sub-cloning, ascites containing $\mathrm{mAb} 3 \mathrm{C} 2$ was generated as previously described, and then the characteristics of mAb $3 \mathrm{C} 2$ were tested. Analysis with a mAb isotyping kit revealed that the isotype of mAb $3 \mathrm{C} 2$ was IgG2b with a Kappa light chain. The ELISA titre of the ascites of mAb $3 \mathrm{C} 2$ could reach 1:1 280000 . mAb 3C2 was also purified using protein $\mathrm{G}$, and only the heavy and light chain could be found in the purified mAb $3 \mathrm{C} 2$, as shown in Figure 1. Specificity analysis showed that $\mathrm{mAb} 3 \mathrm{C} 2$ only reacted with $\mathrm{LMH}$ cells infected with FAdV-4, not with FAdV-8 (Figure 2). Moreover, mAb 3C2 only reacted with LMH cells transfected with pcDNA3.1-Fiber2, not with pcDNA3.1Fiber1 (Figure 2) in IFA. 

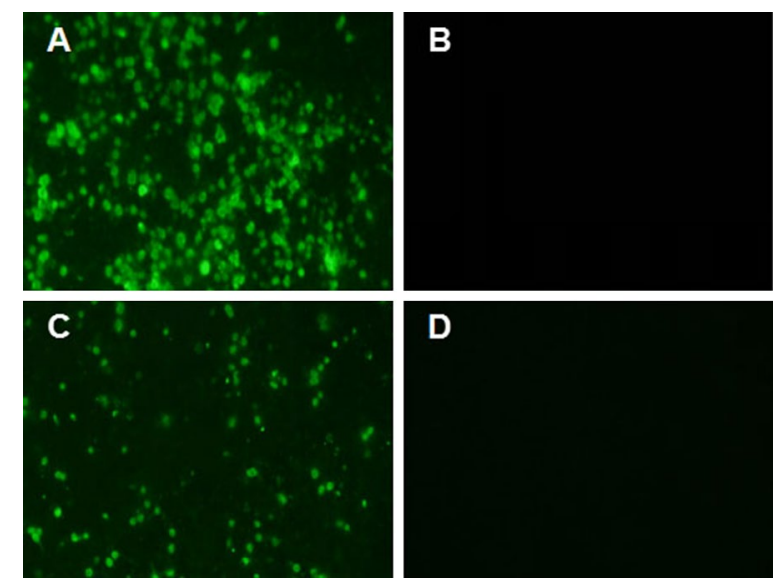

Figure 2 Specificity analysis for $\mathbf{3 C 2}$ by IFA. $L M H$ cells were infected with either FAdV-4 or FAdV-8 or transfected with pcDNA3.1F1 or pcDNA3.1-F2. Then, 3C2 was used as the primary antibody to perform IFA to analyse its specificity. A, B 3 C2 reacted with chicken liver cells infected with FAdV-4 but not FAdV-8. C, D 3 C2 reacted with LMH cells transfected with pcDNA3.1-F2 but not pcDNA3.1-F1.

\section{Immunoprecipitation of the fiber-2 protein of FAdV-4 with $\mathrm{mAb} 3 \mathrm{C} 2$}

Although mAb $3 \mathrm{C} 2$ could efficiently recognize the fiber-2 protein in ELISA and IFA analyses, mAb $3 \mathrm{C} 2$ could not recognize the fiber- 2 protein via Western blot under induced conditions. This indicated that the epitope recognized by $\mathrm{mAb} 3 \mathrm{C} 2$ was likely to be dependent on structure. To further determine whether mAb3 C2 could bind the fiber- 2 protein in cell lysates, immunoprecipitation assays were performed. As shown in Figures $3 \mathrm{~A}$ and $\mathrm{B}$, the purified $\mathrm{mAb} 3 \mathrm{C} 2$ could efficiently immunoprecipitate the fiber-2 protein in LMH cells either infected with FAdV-4 isolate SD2015 or transfected with pcDNA3.1-Fiber2. The immunoprecipitation assay results suggested that $\mathrm{mAb} 3 \mathrm{C} 2$ could be used for identifying the proteins or signaling pathways that the fiber- 2 protein interacts with, thereby elucidating the roles of fiber- 2 in the pathogenesis of FAdV-4.

\section{Inhibition of FAdV-4 infection by mAb $3 \mathrm{C} 2$}

Since the fiber- 2 protein is located on the FAdV-4 viral surface, it might play vital roles in mediating viral infection. To evaluate this hypothesis, we determined whether mAb $3 \mathrm{C} 2$ could block or inhibit the infection or replication of FAdV-4 in vitro. As shown in Figure 4, mAb $3 \mathrm{C} 2$ could efficiently block FAdV-4 infection in a dosedependent manner in neutralization tests. The viral replication of FAdV-4 could not be identified in cells treated with $\mathrm{mAb} 3 \mathrm{C} 2$ at dilutions of $1: 200$ to $1: 800$, whereas FAdV-4 replicated efficiently in cells treated with a control antibody (6E6). Notably, mAb 3C2 treatment at dilutions of 1:1600 to $1: 5000$ still showed efficient blockade of FAdV-4 infection. To further demonstrate the neutralizing activity of mAb $3 \mathrm{C} 2$, Western blot analysis was used to measure the levels of FAdV-4 viral proteins in cells treated with mAb $3 C 2$ at dilutions of 1:200 and 1:5000. As described in Figure 5, virus-specific proteins could not be detected in FAdV-4-infected cells with treatment of mAb $3 \mathrm{C} 2$ at a dilution of 1:200, whereas these viral proteins could be found in FAdV-4-infected cells treated with a control antibody or without any antibody treatment. Moreover, only modest levels of viral proteins could be found in infected cells treated with mAb 3C2 at a dilution of 1:5000. The neutralizing activity of mAb $3 \mathrm{C} 2$

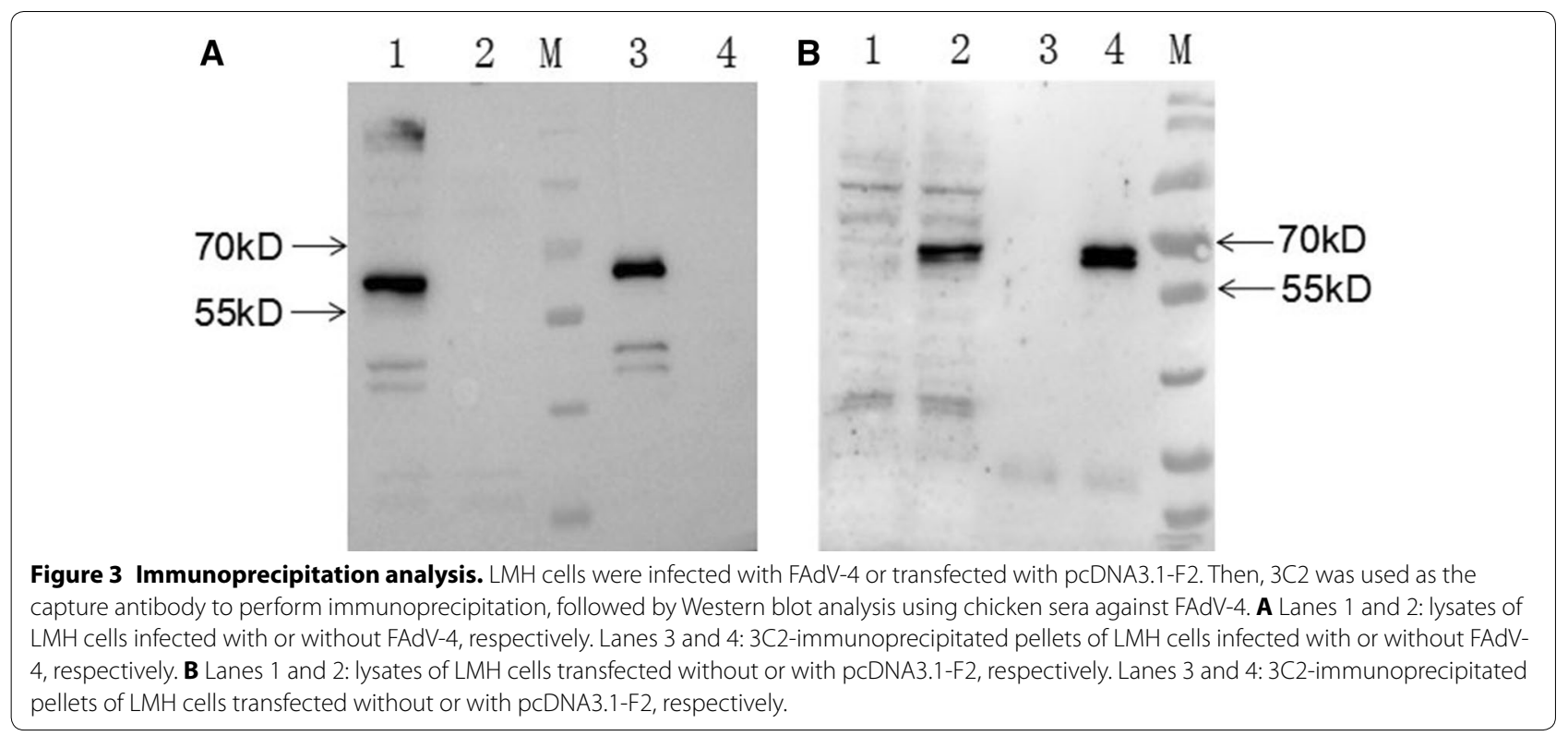




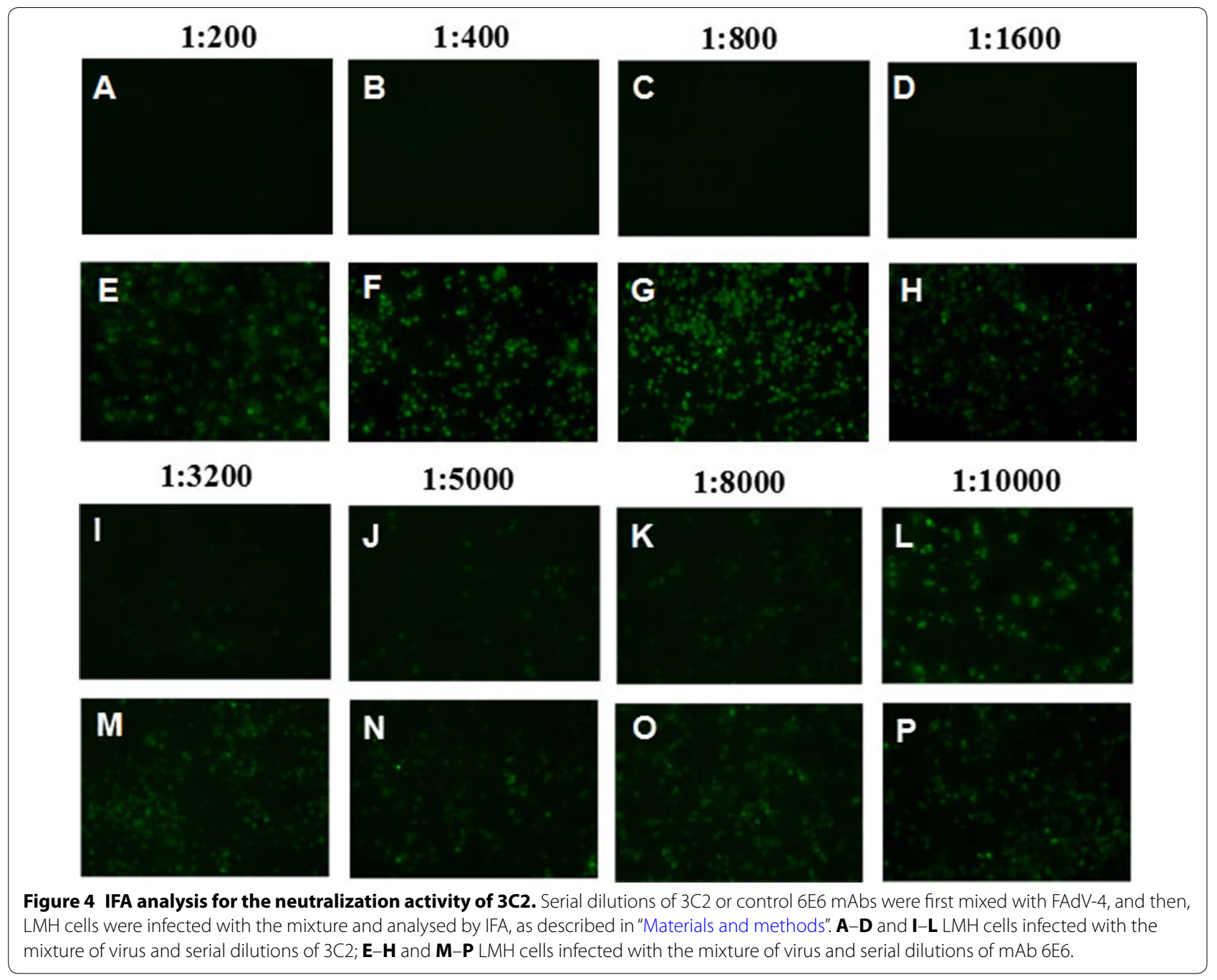

against FAdV-4 indicates that the fiber- 2 protein plays vital roles in mediating the infection of FAdV-4.

\section{Mapping the epitope in fiber-2 recognized by $\mathrm{mAb} 3 \mathrm{C} 2$}

Since mAb 3C2 reacted with a GST-F2_181-448aa fusion protein, but not with a GST-F2_1-180aa fusion protein by ELISA (data not shown), the epitope recognized by mAb 3C2 was located in the C-terminus of F2_181448aa. Therefore, to determine the exact neutralizing epitope in fiber- 2 recognized by $\mathrm{mAb} 3 \mathrm{C} 2$, different truncated fiber- 2 recombinant constructs were transfected into $\mathrm{LMH}$ cells, and IFA was performed. As shown in Figure 6, chicken polyclonal antibodies against FAdV-4 could react robustly with LHM cells transfected with all the different truncated fiber-2 constructs, whereas mAb 3C2 only reacted with LHM cells transfected with full-length fiber-2, not with these truncated fiber- 2 constructs. This demonstrated that the epitope recognized by mAb 3C2 was located at amino acids 416-448 in the
C-terminus of the fiber- 2 protein. Since mAb $3 \mathrm{C} 2$ had high neutralizing activity, the epitope at 416-448aa that reacted with mAb $3 \mathrm{C} 2$ could serve as a novel peptide for designing diagnostic tools or vaccine preparation.

\section{Discussion}

Among the 12 serotypes of FAdV, only serotypes FAdV-1, FAdV-4 and FAdV-10 express two fiber proteins, fiber-1 and fiber-2. Although the vital role of the fiber proteins of FAdV-1 (CELO strain) in viral infection and replication have been demonstrated using CELO mutants expressing truncated fiber- 1 and fiber- 2 proteins, the roles of the fiber proteins of FAdV-4 have not been elucidated. In this study, the critical role of fiber- 2 of FAdV-4 has been demonstrated by the blockade of infection using a novel $\mathrm{mAb}$ $3 \mathrm{C} 2$ targeting fiber- 2 .

A previous report by Schachner et al. demonstrated that fiber-2, but not fiber-1, could provide efficient protection against lethal challenge with FAdV-4 [20]. 


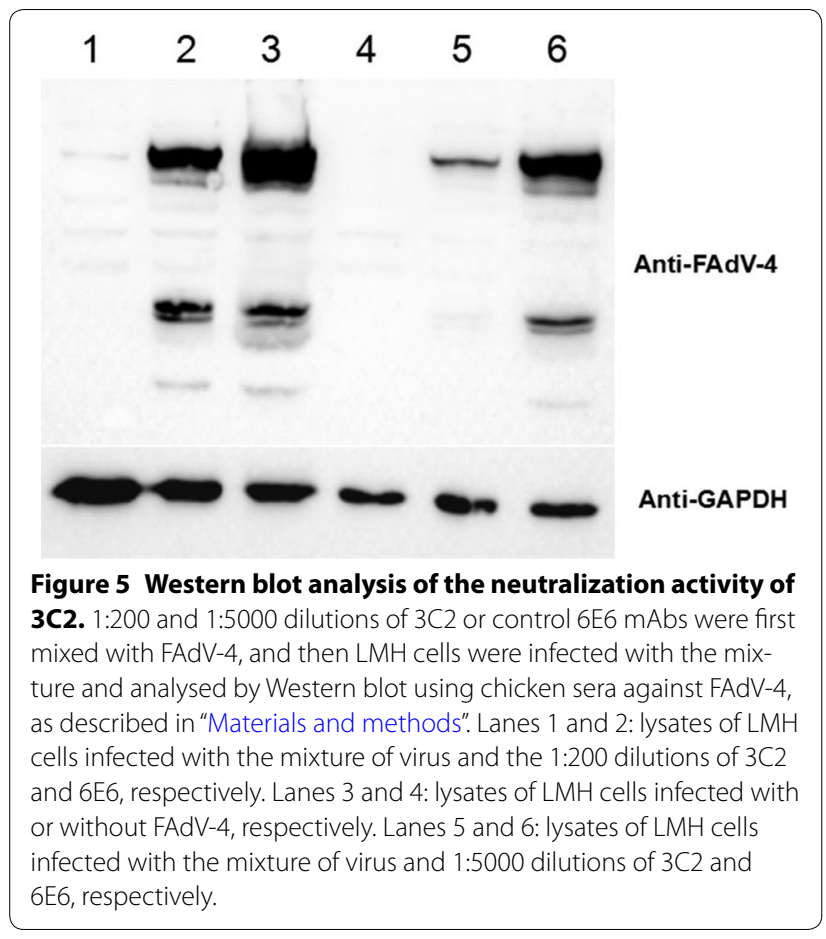

However, neutralizing antibodies in protected chickens prior to challenge could not be efficiently detected by Schachner et al., which indicated that either the fiber-2 expressed by Schachner et al. could not efficiently induce neutralizing antibodies or the neutralizing epitope of the expressed fiber- 2 could not be efficiently targeted by the immune cells. The mAb $3 \mathrm{C} 2$ against fiber- 2 generated here could efficiently block infection by FAdV-4, highlighting that the epitope recognized by $\mathrm{mAb} 3 \mathrm{C} 2$ may be a vital neutralizing epitope. Through the construction of truncated fiber- 2 constructs, the neutralizing epitope recognized by $\mathrm{mAb} 3 \mathrm{C} 2$ was identified to be located between aa $416-448$ in the fiber- 2 protein of FAdV-4. Sequence analysis revealed that four amino acids (at positions 422, 428, 445 and 447) in the epitope showed variation among the different FAdV-4 isolates from the NCBI database. Notably, $47.8 \%$ of FAdV-4 isolates carry an $\mathrm{N}$ residue at position 428 , which provides a potential glycosylation site. The FAdV-4 isolate SD2015 used in this study also has this glycosylation site at position 428. However, the role of this potential glycosylation site at position 428 in the antigenicity and pathogenesis of FAdV-4 remains to be further investigated.

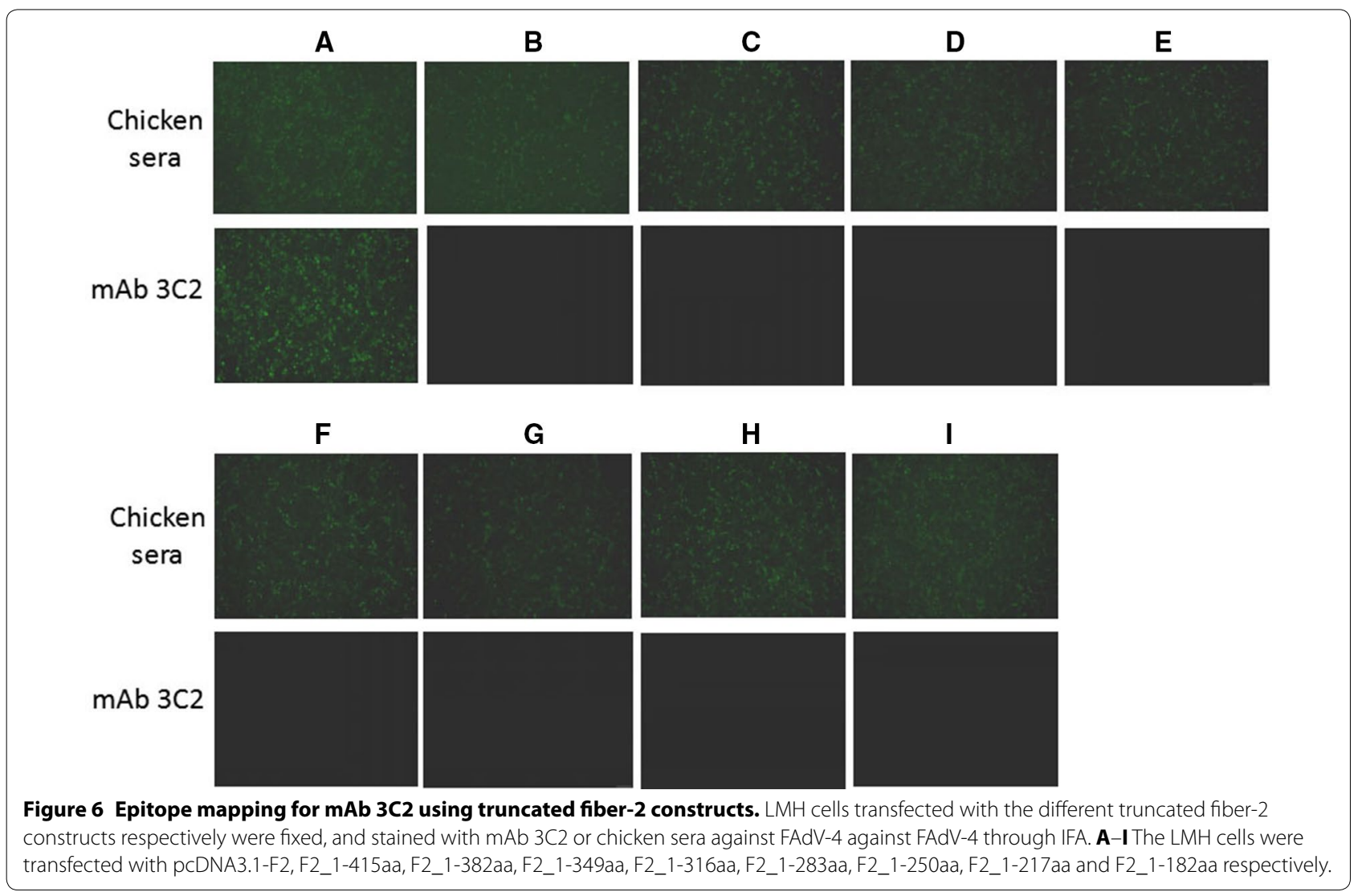


In summary, this is the first demonstration of a novel $\mathrm{mAb}, 3 \mathrm{C} 2$, targeting the fiber- 2 protein of FAdV- 4 with both IP and neutralization activity. mAb $3 \mathrm{C} 2$ specific to the fiber-2 protein could efficiently block infection by FAdV-4, providing strong evidence that the fiber-2 protein plays vital roles in mediating FAdV-4 infection, possibly through binding to its cell receptor. Fiber-2-interacting proteins (including the cell receptor) are currently being identified using $\mathrm{mAb} 3 \mathrm{C} 2$ through Co-IP and Mass Spectrometry analyses. In addition, the $\mathrm{mAb}$ $3 \mathrm{C} 2$ generated here and its identified epitope may be useful for the development of passive immunotherapy and rapid diagnostic tests, such as antigen-capture ELISA or peptide-based ELISA.

\section{Competing interests}

The authors declare that they have no competing interests.

\section{Authors' contributions}

$J Y$ conceived and designed the experiments; PW and JJZ performed the experiments; PW, JZ, AQ, HS, WW and JY analysed the data; JZ,WG, HS and GL contributed reagents/materials/analysis tools; JY, JZ, TL and PW contributed to the writing of the manuscript. PW, WW, TL and JY prepared figures. All authors read and approved the final manuscript.

\section{Acknowledgements}

This work was supported by the National Key Research \& Development (R\&D) Plan (2016YFD0501605, 2016YFD0500803), the National Natural Science Foundation of China (31472171), Key University Science Research Project of Jiangsu Province (14KJA230002), National Science and Technology Transfer FundsProject (2014GB2C100292), Research Foundation for Talented Scholars in Yangzhou University and the Priority Academic Program Development of Jiangsu Higher Education Institutions.

\section{Ethics approval and consent to participate}

All animal experiments were performed in accordance with institutional animal care guidelines, and the protocol, \#06R015, was approved by the Animal Care Committee at Yangzhou University in China.

\section{Author details \\ ${ }^{1}$ Key Laboratory of Jiangsu Preventive Veterinary Medicine, Key Laboratory for Avian Preventive Medicine, Ministry of Education, College of Veterinary Medicine, Yangzhou University, Yangzhou 225009, Jiangsu, China. ${ }^{2}$ Jiangsu Co-innovation Center for Prevention and Control of Important Animal Infec- tious Diseases and Zoonoses, Yangzhou 225009, Jiangsu, China. ${ }^{3}$ Joint Interna- tional Research Laboratory of Agriculture and Agri-Product Safety, The Ministry of Education of China, Yangzhou University, Yangzhou 225009, Jiangsu, China. ${ }^{4}$ Sinopharm Yangzhou VAC Biological Engineering Co. Ltd, Yangzhou 225127, Jiangsu, China. ${ }^{5}$ Institutes of Agricultural Science and Technology Develop- ment, Yangzhou University, Yangzhou 225009, Jiangsu, China.}

\section{Publisher's Note}

Springer Nature remains neutral with regard to jurisdictional claims in published maps and institutional affiliations.

Received: 9 January 2018 Accepted: 26 February 2018

Published online: 09 March 2018

\section{References}

1. Harrach B, Benkö M, Both GW, Brown M, Davison AJ, Echavarría M, Hess M, Jones MS, Kajon A, Lehmkuhl HD, Mautner V, Mittal SK, Wadell G (2012) Family Adenoviridae. In: King AMQ, Adams MJ, Carstens EB, Lefkowitz
EJ (eds) Virus taxonomy, $9^{\text {th }}$ edn. Elsevier Academic Press, San Diego, pp 125-141

2. Niczyporuk JS (2016) Phylogenetic and geographic analysis of fowl adenovirus field strains isolated from poultry in Poland. Arch Virol 161:33-42

3. Mittal D, Jindal N, Tiwari AK, Khokhar RS (2014) Characterization of fowl adenoviruses associated with hydropericardium syndrome and inclusion body hepatitis in broiler chickens. Virusdisease 25:114-119

4. Okuda Y, Ono M, Shibata I, Sato S (2004) Pathogenicity of serotype 8 fowl adenovirus isolated from gizzard erosions of slaughtered broiler chickens. J Vet Med Sci 66:1561-1566

5. De Herdt P, Timmerman T, Defoort P, Lycke K, Jaspers R (2013) Fowl adenovirus infections in Belgian broilers: a ten-year survey. Vlaams Diergen Tijds 82:125-132

6. Kajan GL, Kecskemeti S, Harrach B, Benko M (2013) Molecular typing of fowl adenoviruses, isolated in Hungary recently, reveals high diversity. Vet Microbiol 167:357-363

7. Maartens LH, Joubert HW, Aitchison H, Venter EH (2014) Inclusion body hepatitis associated with an outbreak of fowl adenovirus type 2 and type $8 \mathrm{~b}$ in broiler flocks in South Africa. J S Afr Vet Assoc 85:e1-5

8. Marek AGA, Schulz E, Hess M (2010) Classification of fowl adenoviruses by use of phylogenetic analysis and high-resolution melting-curve analysis of the hexon L1 gene region. JVirol Methods 170:147-154

9. Park HS, Lim IS, Kim SK, Kim TK, Park CK, Yeo SG (2017) Molecular analysis of the hexon, penton base, and fiber-2 genes of Korean fowl adenovirus serotype 4 isolates from hydropericardium syndrome-affected chickens. Virus Genes 53:111-116

10. Choi KS, Kye SJ, Kim JY, Jeon WJ, Lee EK, Park KY, Sung HW (2012) Epidemiological investigation of outbreaks of fowl adenovirus infection in commercial chickens in Korea. Poultry Sci 91:2502-2506

11. Grgic H, Yang DH, Nagy E (2011) Pathogenicity and complete genome sequence of a fowl adenovirus serotype 8 isolate. Virus Res 156:91-97

12. Mase M, Nakamura K, Minami F (2012) Fowl adenoviruses isolated from chickens with inclusion body hepatitis in Japan, 2009-2010. J Vet Med Sci 74:1087-1089

13. Ye J, Liang G, Zhang J, Wang W, Song N, Wang P, Zheng W, Xie Q, Shao H, Wan Z, Wang C, Chen H, Gao W, Qin A (2016) Outbreaks of serotype 4 fowl adenovirus with novel genotype, China. Emerg Microbes Infect $5 \cdot 050$

14. Liu Y, Wan W, Gao D, Li Y, Yang X, Liu H, Yao H, Chen L, Wang C, Zhao J (2016) Genetic characterization of novel fowl aviadenovirus 4 isolates from outbreaks of hepatitis-hydropericardium syndrome in broiler chickens in China. Emerg Microbes Infect 5:e117

15. Zhang T, Jin Q, Ding P, Wang Y, Chai Y, Li Y, Liu X, Luo J, Zhang G (2016) Molecular epidemiology of hydropericardium syndrome outbreak-associated serotype 4 fowl adenovirus isolates in central China. Virol J 13:188

16. Zhao J, Zhong Q, Zhao Y, Hu YX, Zhang GZ (2015) Pathogenicity and complete genome characterization of fowl adenoviruses isolated from chickens associated with inclusion body hepatitis and hydropericardium syndrome in China. PLoS One 10:e0133073

17. Grgic H, Krell PJ, Nagy E (2014) Comparison of fiber gene sequences of inclusion body hepatitis (IBH) and non-IBH strains of serotype 8 and 11 fowl adenoviruses. Virus Genes 48:74-80

18. Henry L, Xia D, Wilke ME, Deisenhofer J, Gerard RD (1994) Characterization of the knob domain of the adenovirus type 5 fiber protein expressed in Escherichia coli. J Virol 68:5239-5246

19. Pallister J, Wright PJ, Sheppard M (1996) A single gene encoding the fiber is responsible for variations in virulence in the fowl adenoviruses. J Virol 70:5115-5122

20. Schachner A, Marek A, Jaskulska B, Bilic I, Hess M (2014) Recombinant FAdV-4 fiber-2 protein protects chickens against hepatitis-hydropericardium syndrome (HHS). Vaccine 32:1086-1092

21. Wan Z, Ye J, Xu L, Shao H, Jin W, Qian K, Wan H, Qin A (2014) Antigenic mapping of the hemagglutinin of an H9N2 avian influenza virus reveals novel critical amino acid positions in antigenic sites. J Virol 88:3898-3901

22. Nelson PN, Reynolds GM, Waldron EE, Ward E, Giannopoulos K, Murray PG (2000) Monoclonal antibodies. Mol Pathol 53:111-117

23. Ye J, Shao H, Hickman D, Angel M, Xu K, Cai Y, Song H, Fouchier RA, Qin A, Perez DR (2010) Intranasal delivery of an IgA monoclonal antibody effective against sublethal H5N1 influenza virus infection in mice. Clin Vaccine Immunol 17:1363-1370 